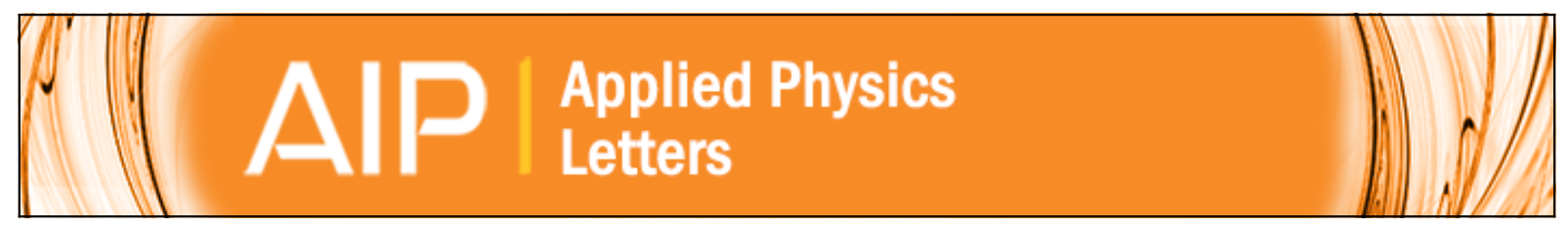

\title{
Magnetic anisotropy obtained from demagnetization curves: Influence of particle orientation and interactions
}

V. Franco and A. Conde

Citation: Applied Physics Letters 74, 3875 (1999); doi: 10.1063/1.124209

View online: http://dx.doi.org/10.1063/1.124209

View Table of Contents: http://scitation.aip.org/content/aip/journal/apl/74/25?ver=pdfcov

Published by the AIP Publishing

\section{Articles you may be interested in}

Multidimensional energy barrier distributions of interacting magnetic particles evaluated at different magnetization states

J. Appl. Phys. 97, 10J315 (2005); 10.1063/1.1853834

Computer simulations of magnetization switching of elongated magnetic particles with shape defects

J. Appl. Phys. 93, 9865 (2003); 10.1063/1.1575494

Magnetic domains in epitaxial nanomagnets with uniaxial or fourfold crystal anisotropy

J. Appl. Phys. 91, 7995 (2002); 10.1063/1.1453339

Interparticle interactions in perpendicularly and longitudinally oriented Ba-ferrite particulate media

J. Appl. Phys. 91, 4418 (2002); 10.1063/1.1453491

From demagnetizing to magnetizing interactions in CoFe-AgCu granular films

J. Appl. Phys. 81, 4593 (1997); 10.1063/1.365173

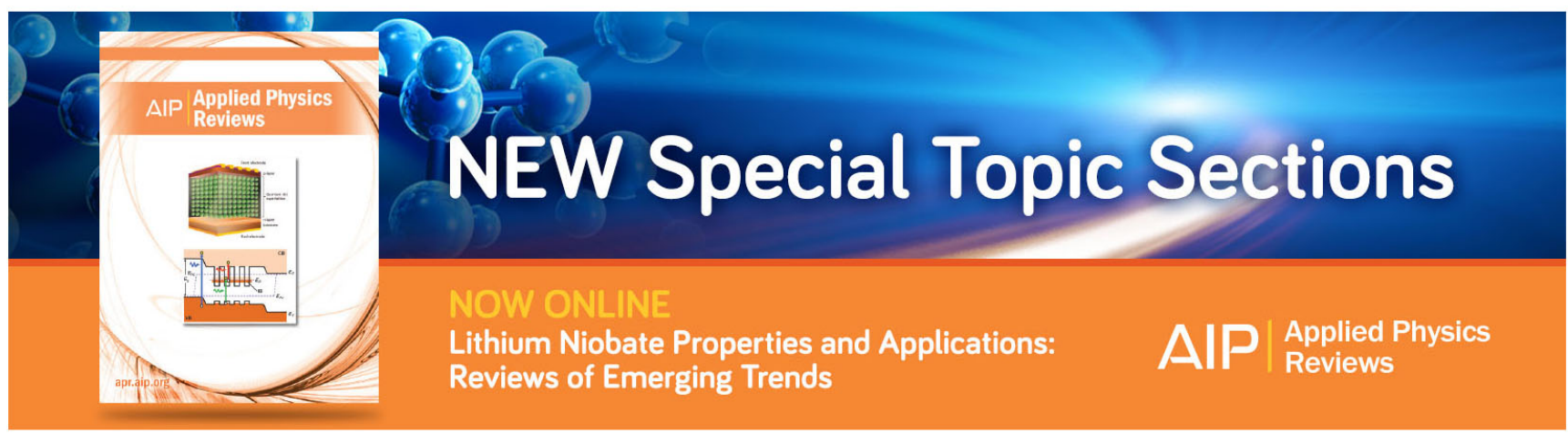




\title{
Magnetic anisotropy obtained from demagnetization curves: Influence of particle orientation and interactions
}

\author{
V. Franco and A. Conde ${ }^{\mathrm{a})}$ \\ Dpto. Física de la Materia Condensada, Instituto de Ciencia de Materiales, C.S.I.C. \\ Universidad de Sevilla, P.O.Box 1065, 41080 Sevilla, Spain
}

(Received 8 March 1999; accepted for publication 27 April 1999)

\begin{abstract}
The influence of easy axis orientation and interparticle interactions on the apparent magnetic anisotropy distribution, obtained from the demagnetization curve analysis, has been studied. For any particle orientation, the method is useful in detecting multimodal distributions as well as effective anisotropy field values. In the case of interacting particulate systems, the range of interaction strength that can be studied is wider than that of the reversible transverse susceptibility method. Analytical expressions for the distribution curves are given, allowing numerical predictions for different distributions of particle orientations and interaction strengths. (C) 1999 American Institute of Physics. [S0003-6951(99)03525-1]
\end{abstract}

Magnetic anisotropy is exploited in the design of most magnetic materials of commercial interest. For hard magnetic materials, the effective anisotropy field is an important parameter in the characterization of their properties, as it gives a theoretical upper limit for coercivity. On the other hand, the magnetic behavior of extremely soft ferromagnetic alloys is being explained by their anisotropy characteristics. ${ }^{1}$ Consequently, it is interesting, both from a theoretical as well as a practical point of view, to dispose of some kind of technique that can give information about the effective anisotropy field of a material, and, if possible, the distribution of anisotropies that gives rise to that effective value.

The magnetic anisotropy distribution can be obtained from a second derivative of the demagnetization curve, ${ }^{2}$ provided that the easy axes are oriented at right angles to the applied magnetic field. This method has been applied to hard magnetic materials ${ }^{3}$ and, recently, to soft nanocrystalline ferromagnetic alloys. ${ }^{4}$ These latter results, where the evolution of the magnetic anisotropy distribution during nanocrystallization of the alloys is presented, are in good agreement with the theoretical predictions of the random anisotropy model extended to multiphase systems. There have also been some studies about compositional effects on the anisotropy distributions, ${ }^{5}$ as well as the relationship between the giant magnetoimpedance (GMI) and these distribution curves. ${ }^{6}$

Despite this amount of experimental work, there is still a lack of a detailed study of the influence of easy axes deviations (or even a distribution of particle orientations) and the effects of interactions between the particles on the effective anisotropy distribution measured by this technique. In this letter we report some theoretical results, based on the Stoner-Wohlfarth model, ${ }^{7}$ concerning the influence of these parameters. It will be proved that, although the shape of the magnetic anisotropy distribution curve is affected by changes in orientation of the easy axes and the possible interactions between the particles, the method is still useful in distinguishing multimodal distributions of anisotropy as well as the effective anisotropy field of the sample.

${ }^{\text {a)} E l e c t r o n i c ~ m a i l: ~ c o n d e @ c i c a . e s ~}$
Based on a Stoner-Wohlfarth model, the distribution of magnetic anisotropy can be obtained from the magnetization curve. Considering the sample under study is composed of a distribution of uniaxial local easy axes with different strengths but all directed at right angles with respect to the applied field, and defining the anisotropy field $H^{K}$ as the one which is necessary to apply in order to saturate an uniaxial particle at right angles to its easy axis, the normalized distribution function of the magnetic anisotropy of the sample is given by

$$
P\left(H^{K}\right)=-H^{K} \frac{d^{2}\langle m\rangle}{d H^{2}},
$$

where $\langle m\rangle$ is the macroscopic reduced magnetization ( $m$ $=M / M_{S}$ ) of the whole ensemble.

In the case of orientations of easy axes that deviates from the $90^{\circ}$ previously considered, the shape of the distribution would be affected. Such a misalignment would produce a broadening of the distribution curve, even for a single value of the anisotropy field. Let's consider a uniaxial particle, with an anisotropy field $H^{K}$, and oriented at an angle $\phi$ with respect to the axis in which the magnetic field is applied. According to Stoner-Wohlfarth, the energy that should be minimized in order to obtain the equilibrium orientation of the magnetization, disregarding constant terms, is

$$
E=1 / 2 \times \sin ^{2}(\phi-\theta)-h \times \cos (\theta),
$$

where $h$ is the reduced applied field $H / H^{K}$ and $\theta$ is the angle between the magnetization and the direction of the applied field $(m=\cos \theta)$.

From the minimum condition, the value of $h$ as a function of the magnetization angle for each particle orientation can be obtained:

$$
h=\sin (\phi-\theta) \times \cos (\phi-\theta) / \sin (\theta) .
$$

To apply Eq. (1), the magnetization curve should be expressed as $\theta(h)$. However, the second derivative of the magnetization with respect to the field can be transformed to an expression in which the different derivatives of Eq. (3) are considered: 


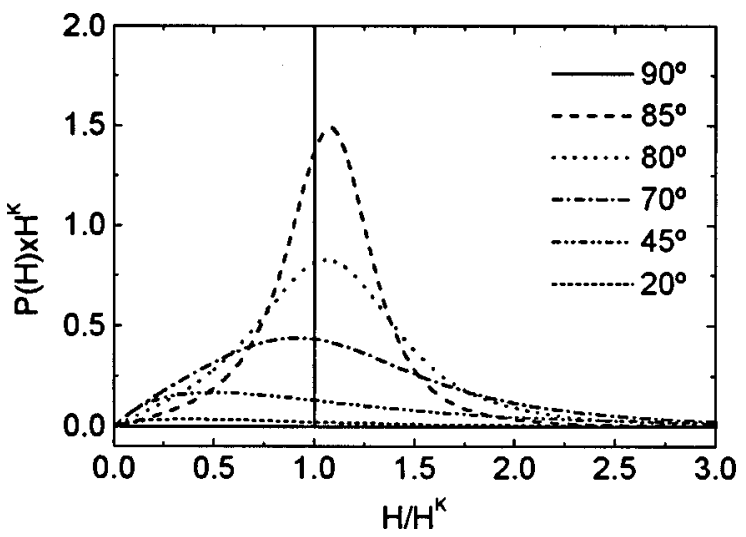

FIG. 1. Influence of particle orientation on the apparent magnetic anisotropy distribution.

$$
\begin{aligned}
& \frac{d^{2} \theta}{d h^{2}}=-\frac{d^{2} h}{d \theta^{2}} \times\left(\frac{d h}{d \theta}\right)^{-3}, \\
& \frac{d^{2} m}{d h^{2}}=\frac{d^{2} \cos (\theta)}{d h^{2}}=\frac{\sin (\theta)}{\left(\frac{d h}{d \theta}\right)^{3}} \times \frac{d^{2} h}{d \theta^{2}}-\frac{\cos (\theta)}{\left(\frac{d h}{d \theta}\right)^{2}} .
\end{aligned}
$$

Combining Eqs. (1), (3), and (5), the probability of obtaining an anisotropy field value of $H$ from the magnetization curve analysis of such a particle is given by

$$
\begin{aligned}
P(H)= & 3 / H^{K} \times \sin (\phi-\theta) \times \cos (\phi-\theta) \times \sin ^{3}(\theta) \\
& \times[\cos (\theta) \times \cos (\theta-\phi)+\sin (\theta-\phi) \times \sin (\theta)] \\
& \times[-\sin (\theta) \times \cos (\theta-\phi)+\cos (\theta) \times \sin (\theta-\phi)] / \\
& {\left[-\cos ^{2}(\theta-\phi) \times \sin (\theta)+\sin ^{2}(\theta-\phi) \times \sin (\theta)\right.} \\
& +\sin (\theta-\phi) \times \cos (\theta-\phi) \times \cos (\theta)]^{3} .
\end{aligned}
$$

In order to present the actual shape of the apparent distributions for different particle orientations, the relations between $\theta$ and $h$, tabulated by Stoner and Wohlfarth, should be used. The results are presented in Fig. 1. As previously stated, for a particle orientation of $90^{\circ}$, the apparent distribution is a Dirac delta at the position of the actual anisotropy field of the particle. Decreasing this angle, the distribution gets wider, indicating a nonexistent anisotropy distribution. The area of the curves diminishes progressively, which means a smaller contribution of the misoriented particles to the changes in magnetization. The behavior of the maximum position is nonmonotonic. Initially, the peak is displaced to higher values of the apparent anisotropy field and, after a critical value, it returns back to lower fields. However, the evolution of the mean anisotropy field obtained from the mean value of the distribution curves is always higher than the actual anisotropy field (except for $90^{\circ}$ ). As a consequence, the smaller error in obtaining the effective anisotropy field in the case of misoriented particles corresponds to the average of the peak position and the mean value of the apparent anisotropy distribution. In that case, the error for any orientation is always below $10 \%$.

If, instead of a single orientation of local easy axes, the system is composed of a distribution of orientations, Eq. (6) should be integrated after multiplying by the proper normalized distribution function. In the case of a random distribu-

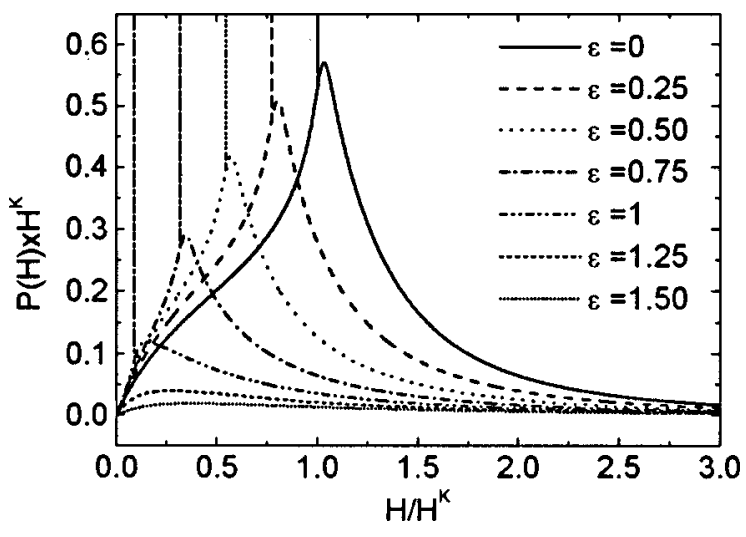

FIG. 2. Influence of the mean field interaction on the apparent anisotropy distribution of a randomly oriented particulate system.

tion of particle orientations (Fig. 2, $\epsilon=0$ ), the peak position would remain at the delta which corresponds to the particles oriented at right angles, while the mean value of the whole distribution is $\left\langle H / H^{K}\right\rangle \sim 1.09$. This means that the error in obtaining the effective anisotropy field would be about $5 \%$ if we consider the previously mentioned method of making the average of the peak position and the mean value. It is clear that, although the apparent distribution curve is distorted because of the changes in particle orientations, the curve can be useful in detecting multimodal distributions.

Many real systems are composed of an ensemble of interacting particles, which cannot be analyzed by the method studied so far. Thus, it is important to consider the possible influences that the interactions can have on the apparent anisotropy distribution.

To represent the interactions between the particles, the mean field approach proposed by Callen et al. ${ }^{8}$ has been used. The effective field that acts upon any particle in the system is $H_{e}=H+\lambda M$, where $\lambda$ is the parameter that controls the interaction and $M$ the magnetization of the whole ensemble. Although the mean field approach is not suitable for calculating coercivities, as for calculating the magnetic anisotropy distribution the magnetization curve from saturation to remanence is only needed, this kind of interaction can be used as a first approach to the problem.

Transforming to adimensional variables, the effective field is $h_{e}=h+\epsilon m$, where $\epsilon=\lambda M_{s} / H^{K}$. Considering a random distribution of local easy axes, the magnetization curve should be calculated using a self-consistent procedure. Once this magnetization curve is obtained, the easiest way to obtain the apparent anisotropy distribution is to directly apply Eq. (1). However, this procedure gives a distribution curve with a huge amount of noise, due to the numerical derivatives, which makes it difficult to clarify if some of the features of the curve are artifacts or not.

On the contrary, we have chosen the method of composing the anisotropy curve of the ensemble from the contributions of the individual particles [Eq. (6)]. To do that, it is necessary to take into account that the only effect of the interactions is to alter the effective field that acts on each particle. With that in mind, it is clear that the hysteresis loops of the differently interacting systems, once represented versus $h_{e}$ instead of $h$, should overlap (apart from some differences in the stability of the different regions of the loop). 
Transforming Eq. (5) to this effective field coordinate system, it can be proven that ${ }^{9}$

$$
\frac{d^{2} m}{d h^{2}}=\frac{d^{2} m}{d h_{e}^{2}} \times\left(1-\varepsilon \frac{d m}{d h_{e}}\right)^{-3},
$$

where the derivatives with respect to $h_{e}$ are the same than those of the noninteracting system.

In Fig. 2, the evolution with the interaction parameter of the apparent anisotropy distribution of a randomly oriented particulate system is presented. It is clearly seen that the influence of this mean field interaction is to displace the distribution to lower anisotropy fields, a feature that, together with the increase of remanence, is analogous to what would be produced by a rotation of all the particles of the system to orientations closer to the applied field. At the same time, the Dirac delta approaches the $h=0$ axis and, after the interaction reaches the value necessary to place that delta over the OY axis, its contribution disappears. From that value of $\epsilon$ ( $\left.\epsilon_{\text {crit }} \sim 1.095\right)$, the particles that would contribute to the anisotropy distribution curve are those whose nucleation field is progressively increased (in $h_{e}$ coordinates). In the region where $\epsilon<\epsilon_{\text {crit }}$, the evolution of the distribution peak is linear (Fig. 3). This result is similar to that reported for the determination of the effective anisotropy field from transverse suscepibility measurements, ${ }^{10}$ but, in our case, the linearity region is doubled. For a higher interaction strength, the behavior of the characteristic parameters of the distribution is no longer linear, due to the changes in the particles that are contributing to the curve, and the smaller error in the determination of the anisotropy field is obtained from the average of the peak position and the mean value of the distribution.

An alternative way of obtaining information of the anisotropy of a sample is the approach to saturation. ${ }^{11}$ Under the assumptions of a random distribution of uniaxial local easy axes, the magnetization curve in the approach to the saturation region can be represented by ${ }^{12} m(H)=1-b / H^{2}$, with $b=1 / 15 \times\left(H^{K}\right)^{2}$. Consequently, the apparent distribution of anisotropy should correspond to

$$
P(H)=6 / 15 \times\left(H^{K}\right)^{2} \times H^{-3} .
$$

To check the applicability of this expression to the interacting system, the apparent distributions, for $\epsilon$ values such that all the particles in the system contribute to the anisotropy curve (i.e., $\epsilon<\epsilon_{\text {crit }}$ ) and an anisotropy field of $H^{K}=1$, have been fitted to the function $P(H)=A \times\left(H-H_{0}\right)^{C}$ by means of the Levenberg-Marquardt algorithm. ${ }^{13}$ The term $H_{0}$ has been included to take into account the observed effect of the displacement of the anisotropy curves due to the change in the interaction strength. The fitting procedure gave the same values for the prefactor and the exponent for all the curves $(A=0.40078 \sim 6 / 15 ; C=-2.99686 \sim-3)$ and a linear decrease of $H_{0}$ as the interaction parameter is increased, with a slope of $-1.37\left[H_{0} / \epsilon\right]$. From these results, it is clear that the region of approach to saturation in the magnetic anisotropy distribution curve can be used to obtain informa-

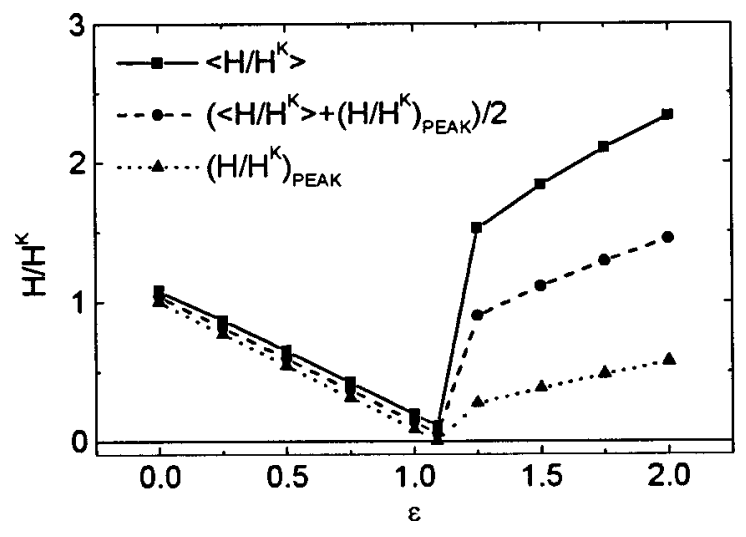

FIG. 3. Evolution of the apparent anisotropy distribution characteristic parameters with the value of interaction strength.

tion about the effective anisotropy field (parameter $A$ ) as well as the magnitude of the interactions (from $H_{0}$ ).

In conclusion, the analytical expressions of the apparent magnetic anisotropy distribution have been given for any orientation of the easy axis, which gives the possibility of computing the predicted curves for any orientational distribution in a particulate system. The influence of interparticle interactions has also been analyzed by means of a mean field approach. These results indicate that the range of interaction strength for which anisotropy information can be obtained is broader than that allowed by the transverse susceptibility method. The method is straightforward in obtaining anisotropy distributions for systems in which the easy axes are oriented at $90^{\circ}$ with respect to the applied field, but, in other cases, a more careful analysis of the distribution curves should be employed, although there would be no confusion in detecting multimodal distributions.

This work was supported by the DGES of the Spanish Ministry of Education (Project PB97-1119-CO2-01). V. Franco is grateful to the Fundación Cámara of Sevilla University for a research fellowship.

\footnotetext{
${ }^{1}$ A. Hernando, M. Vázquez, T. Kulik, and C. Prados, Phys. Rev. B 51, 3581 (1995); A. Hernando, P. Marín, M. Vázquez, J. M. Barandiarán, and G. Herzer, Phys. Rev. B 58, 366 (1998); K. Suzuki and J.M. Cadogan, Phys. Rev. B 58, 2730 (1998); J. Arcas, A. Hernando, J. M. Barandiarán, C. Prados, M. Vázquez, P. Marín, and A. Neuweiler, Phys. Rev. B 58, 5193 (1998).

${ }^{2}$ J. M. Barandiarán, M. Vázquez, A. Hernando, J. González, and G. Rivero, IEEE Trans. Magn. 25, 3330 (1989).

${ }^{3}$ G. Bottoni, D. Candolfo, and A. Cecchetti, J. Appl. Phys. 81, 3794 (1997).

${ }^{4}$ V. Franco, C. F. Conde, and A. Conde, J. Magn. Magn. Mater. 185, 353 (1998).

${ }^{5}$ V. Franco, C. F. Conde, and A. Conde, J. Appl. Phys. 84, 5108 (1998).

${ }^{6}$ V. Franco and A. Conde (unpublished).

${ }^{7}$ E.C. Stoner and E. P. Wohlfarth, Proc. R. Soc. London, Ser. A 240, 599 (1948).

${ }^{8}$ E. Callen, Y. J. Liu, and J. R. Cullen, Phys. Rev. B 16, 263 (1977).

${ }^{9}$ V. Franco, Ph.D. thesis, Sevilla University, 1999.

${ }^{10}$ C. R. Chang and J. S. Yang, Appl. Phys. Lett. 65, 496 (1994).

${ }^{11}$ S. Chikazumi, Physics of Magnetism (Krieger, Malabar, 1978).

${ }^{12}$ K. Y. Ho, X. Y. Xiong, J. Zhi, and L. Z. Cheng, J. Appl. Phys. 74, 6788 (1993).

${ }^{13}$ D. W. Marquardt, J. Soc. Ind. Appl. Math. 11, 431 (1963).
} 